\title{
Disconjugacy and the Secant Conjecture
}

\author{
Alexandre Eremenko ${ }^{1}$
}

Received: 5 July 2015 / Accepted: 28 July 2015 / Published online: 4 August 2015

(C) Institute for Mathematical Sciences (IMS), Stony Brook University, NY 2015

\begin{abstract}
We discuss the so-called secant conjecture in real algebraic geometry, and show that it follows from another interesting conjecture, about disconjugacy of vector spaces of real polynomials in one variable.
\end{abstract}

Keywords Disconjugacy $\cdot$ Wronskian $\cdot$ Schubert calculus

Mathematics Subject Classification $14 \mathrm{P} 99 \cdot 34 \mathrm{C} 10 \cdot 26 \mathrm{C} 10$

Let $V$ be a real vector space of dimension $n$ whose elements are real functions on an interval $[a, b]$. The space $V$ is called disconjugate if one of the following equivalent conditions is satisfied:

(a) Every $f \in V \backslash\{0\}$ has at most $n-1$ zeros, or

(b) For every $n$ distinct points $x_{1}, \ldots, x_{n}$ on $[a, b]$ and every basis $f_{1}, \ldots, f_{n}$ of $V$ we have $\operatorname{det}\left(f_{i}\left(x_{j}\right)\right) \neq 0$.

One can replace "every basis" by "some basis" in (b) and obtain an equivalent condition.

If $V$ is disconjugate then the determinant in (b) has constant sign which depends only on the ordering of $x_{j}$ and on the choice of the basis.

A space of real functions on an open interval is called disconjugate if it is disconjugate on every closed subinterval.

Supported by NSF grant DMS-1361836.

$\bowtie \quad$ Alexandre Eremenko

eremenko@math.purdue.edu

1 Department of Mathematics, Purdue University, West Lafayette, IN 47907, USA 
We are only interested here in spaces $V$ consisting of polynomials.

Suppose that a positive integer $d$ is given, and $V$ consists of polynomials of degree a most $d$. Then every basis $f_{1}, \ldots, f_{n}$ of $V$ defines a real rational curve $\mathbf{R P}^{1} \rightarrow \mathbf{R} \mathbf{P}^{n-1}$ of degree $d$. Indeed, we can replace every $f_{j}(x)$ by a homogeneous polynomial $f_{j}^{*}\left(x_{0}, x_{1}\right)$ of two variables of degree $d$, such that $f_{j}(x)=f_{j}^{*}(1, x)$, and then $f_{1}^{*}, \ldots, f_{n}^{*}$ define a map $f: \mathbf{R P}^{1} \rightarrow \mathbf{R} \mathbf{P}^{n-1}$ (if polynomials have a common root, divide it out).

Then the geometric interpretation of disconjugacy is:

(c) The curve $f$ constructed from a basis in $\nabla$ is convex, that is intersects every hyperplane at most $n-1$ times.

For every basis $f_{1}, \ldots, f_{n}$ in $V$ we can consider its Wronski determinant $W=$ $W\left(f_{1}, \ldots, f_{n}\right)$. Changing the basis results in multiplication of $W$ by a non-zero constant, so the roots of $W$ only depend on $V$.

Conjecture 1 Suppose that all roots of $W$ are real. Then $V$ is disconjugate on every interval that does not contain these roots.

This is known for $n=2$ with arbitrary $d$ (see below), and for $n=3, d \leq 5$ by direct verification with a computer.

This conjecture arises in real enumerative geometry (Schubert calculus), and we explain the connection. The problem of enumerative geometry we are interested in is the following:

Let $m \geq 2$ and $p \geq 2$ be given integers. Suppose that mp linear subspaces of dimension $p$ in general position in $\mathbf{C}^{m+p}$ are given. How many linear subspaces of dimension $m$ intersect all of them non-trivially?

The answer was obtained by Schubert in 1886 and it is

$$
d(m, p)=\frac{1 ! 2 ! \ldots(p-1) !(m p) !}{m !(m+1) ! \ldots(m+p-1) !} .
$$

Now suppose that all those given subspaces are real. Does it follow that all $p$-subspaces that intersect all of them non-trivially are real? The answer is negative, and we are interested in finding a geometric condition on the given $p$-subspaces that ensure that all $d(m, p)$ subspaces of dimension $m$ that intersect all the given $p$-subspaces nontrivially are real.

One such condition was proposed by B. and M. Shapiro. Let $F(x)=\left(1, x, \ldots, x^{d}\right)$, $d=m+p-1$ be a rational normal curve, a. k. a. moment curve. Suppose that the given $p$-spaces are osculating $F$ at some real points $F\left(x_{j}\right)$. This means that subspaces $X_{j}$ are spanned by the (row)-vectors $F\left(x_{j}\right), F^{\prime}\left(x_{j}\right), \ldots, F^{(p-1)}\left(x_{j}\right)$ for some real $x_{j}, 1 \leq j \leq m p$. Then all $m$-subspaces that intersect all $X_{j}$ non-trivially are real.

This was conjectured by B. and M. Shapiro and proved by Mukhin, Tarasov and Varchenko (MTV) Mukhin et al. (2009). Earlier it was known for $n=2$ Eremenko and Gabrielov (2002), and in Eremenko and Gabrielov (2011) a simplified elementary proof for the case $n=2$ was given.

We are interested in the following generalization of this result.

Secant Conjecture. Suppose that each of the mp subspaces $X_{j}, 1 \leq j \leq m p$ is spanned by $p$ distinct real vectors $F\left(x_{j, k}\right), 0 \leq k \leq p-1$, and that the sets of points 
$\left\{x_{j, k}: 0 \leq k \leq p-1\right\}$ are separated, that is $x_{j, k} \in I_{j}$, where $I_{j} \subset \mathbf{R P}^{1}$ are disjoint intervals. Then all $m$-subspaces which intersect all $X_{j}$ non-trivially are real.

This is known when $p=2$, Eremenko et al. (2006) and has been tested on a computer for $p=3$ and small $m$, Hillar et al. (2010), Garcia-Puente et al. (2012). The special case when the groups $\left\{x_{j, k}\right\}_{k=0}^{p-1}$ form arithmetic progressions, $x_{j, k}=x_{j, 0}+k h$ has been established Mukhin et al. (2009).

Next we show how the Secant Conjecture follows from Conjecture 1 and the results of MTV.

Let us represent an $m$-subspace $Y$ that intersects all subspaces $X_{j}$ as the zero set of $p$ linear forms, and use the coefficients of these forms as coefficients of $p$ polynomials $f_{0}, \ldots, f_{p-1}$. Then the condition that $Y$ intersects some $X_{j}$ is equivalent to linear dependence of the $p$ vectors $f_{i}\left(x_{j, m}\right)_{m=0}^{p-1}, i=0, \ldots, p-1$. That is

$$
\operatorname{det}\left(f_{i}\left(x_{j, m}\right)\right)_{i, m=0}^{p-1}=0 .
$$

These equations for $j=1, \ldots, m p$ define the subspaces $Y$, and we have to prove that all solutions are real.

Let $I_{j}$ be the intervals with disjoint closures which contain the $x_{j, k}$. We may assume without loss of generality that $\infty \notin I_{j}$. We place on each $I_{j}$ a point $y_{j}$, and consider the $d(m, p)$ real rational curves $\mathbf{R} \rightarrow \mathbf{R P}^{p}$ with inflection points at $y_{j}$. These curves exist by the MTV theorem, and they depend continuously on the $y_{j}$.

Let $f=\left(f_{0} \ldots, f_{p-1}\right)$ be one of these curves. Fix $k \in\{1, \ldots, m p\}$. For all $j \neq k$, fix all $y_{j} \in I_{j}$. When $y_{k}$ moves in $I_{k}$ from the left end to the right end, the determinant $\operatorname{det}\left(f_{i}\left(x_{k, m}\right)\right)_{i, m=0}^{p-1}$ must change sign, in view of Conjecture 1 . So this determinant is 0 for some position of $y_{k}$ on $I_{k}$.

Then it follows by a well-known topological argument that one can choose all $y_{j} \in I_{j}$ in such a way that $\operatorname{det}\left(f_{i}\left(x_{j, m}\right)\right)=0$ for all $j$.

Thus we have constructed $d(m, p)$ real solutions of the secant problem. As the total number of solutions is also $d(m, p)$, for generic data, we obtain the result.

Proof of Conjecture 1 for $n=2$. We have two real polynomials $f_{1}$ and $f_{2}$, such that $f_{1}^{\prime} f_{2}-f_{1} f_{2}^{\prime}$ has only real zeros. This means that the rational function $F=f_{1} / f_{2}$ : $\overline{\mathbf{C}} \rightarrow \overline{\mathbf{C}}$ is real and all its critical points are real. Let $I \subset \mathbf{R}$ be a closed interval without critical points. Then $F$ is a local homeomorphism on $I$, so $F(I+i \epsilon)$ belongs to one of the half-planes $\mathbf{C} \backslash \mathbf{R}$, for all sufficiently small $\epsilon>0$. Suppose without loss of generality that it belongs to the upper half-plane $H$. Let $D$ be the component of $F^{-1}(H)$ that contains $I+i \epsilon$. Then $D$ is a region in $H$ with piecewise analytic boundary, and $I \subset \partial D$. The map $F: D \rightarrow H$ is a covering because it is proper and has no critical points. As $H$ is simply connected, $D$ must be simply connected and $F: D \rightarrow H$ must be a conformal homeomorphism. Then $F^{-1}: H \rightarrow D$ is a conformal homeomorphism. As $\partial D$ is locally connected, this homeomorphism extends to $F^{-1}: \bar{H} \rightarrow \bar{D}$. This last map must be injective because this is a left inverse of a function. Thus $F^{-1}: \bar{H} \rightarrow \bar{D}$ is a homeomorphism. Then $F: \bar{D} \rightarrow \bar{H}$ must be also a homeomorphism, in particular $F$ is injective on $I$.

This implies that the linear span of $f_{1}, f_{2}$ is disconjugate. 


\section{References}

Eremenko, A., Gabrielov, A.: Rational functions with real critical points and the B. and M. Shapiro conjecture in real enumerative geometry. Ann. Math. 155, 105-129 (2002)

Eremenko, A., Gabrielov, A.: Elementary proof of the B. and M. Shapiro conjecture for rational functions. In: Notions of Positivity and the Geometry of Polynomials, Trends in Mathematics, pp. 167-178. Springer, Basel (2011)

Eremenko, A., Gabrielov, A., Shapiro, M., Vainshtein, A.: Rational functions and real Schubert calculus. Proc. AMS 134(4), 949-957 (2006)

Garcia-Puente, L., Hein, N., Hillar, Ch., del Campo, A., Ruffo, J., Sottile, F., Teitler, Z.: The Secant Conjecture in the real Schubert calculus. Exp. Math. 21(3), 252-265 (2012)

Hillar, C., Garcia-Puente, L., Martin del Campo, A., Ruffo, J., Teitler, Z., Johnson, S., Sottile, F.: Experimentation at the Frontiers of Reality in Schubert Calculus. Gems in Experimental Mathematics, pp. 365-380. Contemporary Mathematics, vol. 517. American Mathematical Society, Providence (2010)

Mukhin, E., Tarasov, V., Varchenko, A.: The B. and M. Shapiro conjecture in real algebraic geometry and the Bethe ansatz. Ann. Math. 170(2), 863-881 (2009)

Mukhin, E., Tarasov, V., Varchenko, A.: On reality property of Wronski maps. Conflu. Math. 1, 225-247 (2009) 